\title{
A Compactification of the Space of Expanding Maps on the Circle
}

\section{Citation}

McMullen, Curtis T. 2009. A compactification of the space of expanding maps on the circle. Geometric and Functional Analysis 18(6): 2101-2119.

\section{Published Version}

http://dx.doi.org/10.1007/s00039-009-0709-8

\section{Permanent link}

http://nrs.harvard.edu/urn-3:HUL.InstRepos:3426329

\section{Terms of Use}

This article was downloaded from Harvard University's DASH repository, and is made available under the terms and conditions applicable to Open Access Policy Articles, as set forth at http:// nrs.harvard.edu/urn-3:HUL.InstRepos:dash.current.terms-of-use\#OAP

\section{Share Your Story}

The Harvard community has made this article openly available.

Please share how this access benefits you. Submit a story.

Accessibility 


\title{
A compactification of the space of expanding maps on the circle
}

\author{
Curtis T. McMullen*
}

2 July, 2007

\begin{abstract}
We show the space of expanding Blaschke products on $S^{1}$ is compactified by a sphere of invariant measures, reminiscent of the sphere of geodesic currents for a hyperbolic surface. More generally, we develop a dynamical compactification for the Teichmüller space of all measure-preserving topological covering maps of $S^{1}$.
\end{abstract}

\section{Contents}

1 Introduction . . . . . . . . . . . . . . . 1

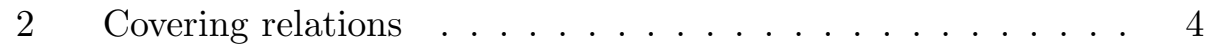

3 Blaschke products . . . . . . . . . . . . . 13

4 Polynomials and harmonic measure . . . . . . . . . . . . 15

\section{Introduction}

Let $\operatorname{Exp}_{d}\left(S^{1}\right)$ denote the space of topological covering maps $f: S^{1} \rightarrow S^{1}$ of degree $d>1$ that preserve Lebesgue measure. Such a map is expanding, in the sense that $|f(I)|>|I|$ for any interval where $f \mid I$ is injective.

In this paper we introduce a compactification $\widehat{\operatorname{Exp}}_{d}\left(S^{1}\right)$ for the space of expanding maps, generalizing the compactification of rational maps $f$ : $\mathbb{R P}^{1} \rightarrow \mathbb{R P}^{1}$ by algebraic correspondences. The limiting objects in the compactification are covering relations, which are allowed to blow up single points to intervals and wrap them around the circle.

It is well-known that every $f \in \operatorname{Exp}_{d}\left(S^{1}\right)$ is topologically conjugate to the model mapping $p_{d}(t)=d \cdot t \bmod 1$ (see e.g. [Sh], [Fra] for the $C^{1}$ case). There are $(d-1)$ choices for conjugacy $\phi$; the choice of one is a marking of

${ }^{*}$ Research supported in part by the NSF.

2000 Mathematics Subject Classification: Primary 37F30, Secondary 37A05, 37E10. 
$f$. Using the marking, one can transport Lebesgue measure to an invariant measure $\nu_{(f, \phi)}=\phi_{*}(\lambda)$ for the model mapping $p_{d}$.

In $\S 2$ we extend this construction to the covering relations, and establish:

Theorem 1.1 The map

$$
\nu: \widehat{\operatorname{Exp}}_{d}\left(S^{1}\right) \rightarrow M_{d}\left(S^{1}\right)
$$

gives a bijective homeomorphism between the compactified Teichmüller space of expanding maps and the space of invariant probability measures for $t \mapsto$ $d \cdot t \bmod 1$.

In particular, we construct an expanding dynamical system $f: S^{1} \rightarrow S^{1}$ for every $p_{d}$-invariant measure $\nu$ on the circle.

Using this result, we show $\operatorname{TExp}_{d}\left(S^{1}\right)$ is dense in its compactification $\widehat{\operatorname{Exp}}_{d}\left(S^{1}\right)$, and both spaces are contractible. Along the way we show every $f \in \widehat{\operatorname{Exp}}_{d}\left(S^{1}\right)$ has a natural measure of maximum entropy $\mu_{f}$, which varies continuously with $f$. An analogous result for rational maps and algebraic correspondences appears in [D1, Thm. 0.1].

Blaschke products. Next we specialize to the case where $f \in \operatorname{Exp}_{d}\left(S^{1}\right)$ is a rational map on $S^{1} \cong \mathbb{R} \mathbb{P}^{1}$ of algebraic degree $d$; equivalently, where $f$ extends holomorphically to the unit disk $\Delta$ in the complex plane. Then up to conjugation by a rotation, $f$ has the form

$$
f(z)=z \prod_{1}^{d-1}\left(\frac{z-a_{i}}{1-\bar{a}_{i} z}\right)
$$

$\left|a_{i}\right|<1$. Every such map preserves Lebesgue measure and has a natural marking, determining an inclusion $\mathcal{B}_{d} \hookrightarrow \operatorname{TExp}_{d}$.

Allowing the points $\left(a_{i}\right)$ to go to the circle, we obtain a compactification $\overline{\mathcal{B}}_{d} \cong \bar{\Delta}^{(d-1)}$. An element $f \in \overline{\mathcal{B}}_{d}$ is given by a pair $(F, S)$ consisting of a Blaschke product $F$ and a divisor of sources, $S=\sum m_{i} s_{i} \in \operatorname{Div}\left(S^{1}\right)$, satisfying $\operatorname{deg}(F)+\operatorname{deg}(S)=d$.

In $\S 3$ we show these pairs $(F, S)$ can be naturally identified with covering relations, and establish:

Theorem 1.2 The map $\nu: \overline{\mathcal{B}}_{d} \rightarrow M_{d}\left(S^{1}\right)$ is an embedding.

Corollary 1.3 The boundary of $\nu\left(\mathcal{B}_{d}\right)$ in the space of invariant measures $M_{d}\left(S^{1}\right)$ is a sphere of dimension $(2 d-3)$. 
Currents. One can compare the compactification of $\mathcal{B}_{d}$ by invariant measures for $z^{d}$ to the compactification of Teichmüller space by measured laminations, thought of as invariant measures for the geodesic flow [FLP], [Bon].

Strata. In $\S 3$ we show that the natural strata of $\overline{\mathcal{B}}_{d}$, defined by $\operatorname{deg}(F)=e$, are visible at the level of invariant measures as well.

Theorem 1.4 The locus $\Delta^{(e-1)} \times\left(S^{1}\right)^{(d-e)}$ in $\partial \mathcal{B}_{d}$ maps to measures satisfying

$$
\text { H. } \operatorname{dim} \operatorname{supp} \nu_{f}=\frac{\log e}{\log d} \text {. }
$$

In particular, certain invariant measures for $z^{d}$ are succinctly encoded by maps $f=(F, S) \in \partial \mathcal{B}_{d}$. See $\S 4$ for the examples $(F, S)=(\lambda z,-\lambda)$ and $\left(z^{2},-1\right)$, which give ergodic invariant measures supported on Cantor sets of dimension zero and $\log 2 / \log 3$ respectively.

Rotations sets. A rotation set $C \subset S^{1}$ is a finite invariant set whose cyclic order is preserved by $p_{d}$. These sets, which are classified in [Gol], can always be given in the form

$$
C=\operatorname{supp} \nu_{((F, S), \phi)},
$$

where $F(t)=t+p / q \bmod 1$ is a periodic rotation. Conversely, as the divisor $S$ varies, all $C$ with rotation number $p / q$ arise. This labeling of rotation sets by divisors provides an alternative approach to their combinatorics, as well as an extension to degrees $e>1$ (by taking $F(t)=e \cdot t \bmod 1$ ).

Polynomials and harmonic measure. We conclude by formulating a relationship between covering relations and Julia sets of polynomials.

Let $P(z)=z^{d}+b_{1} z^{d-1}+\cdots+b_{d}$ be a monic polynomial of degree $d>1$, such that $P(0)=0$, the filled Julia set $K(P)$ is locally connected, and $z=0$ lies in a component $U_{0}(P)$ of the interior of $K(P)$.

Let $K_{0}(P)=\overline{U_{0}(P)}$ and let $J_{0}(P)=\partial K_{0}(P)$. There is a unique retraction $\rho: K(P) \rightarrow K_{0}(P)$ which is locally constant outside its image. Using the Riemann mapping theorem, one can transfer $P \mid K_{0}(P)$ to a Blaschke product $F \mid \bar{\Delta}$, together with a natural divisor $S \in \operatorname{Div}\left(S^{1}\right)$ determined by the critical points of $P$ outside $U_{0}$.

Let $\mu_{P}$ and $\nu_{P}$ denote the hitting measures on $J(P)=\partial K(P)$ for Brownian motion started at $z=\infty$ and $z=0$ respectively. In $\S 4$ we will show:

Theorem 1.5 For any polynomial $P$ as above,

1. The parameterization of $J_{0}(P)$ by internal angles sends $\mu_{(F, S)}$ to $\rho_{*}\left(\mu_{P}\right)$; 
2. The retraction $\rho: K(P) \rightarrow K_{0}(P)$ gives a marking $\phi$ for $(F, S)$; and

3. The parameterization of $J(P)$ by external angles sends $\nu_{((F, S), \phi)}$ to $\nu_{P}$.

Corollary 1.6 The external angles of points in $J_{0}(P)$ form a subset of the circle of dimension $\log e / \log d$, where $e=\operatorname{deg}\left(P \mid U_{0}(P)\right)$.

Proof. This set of angles agrees with $\operatorname{supp} \nu_{((F, S), \phi)}$.

For more details and examples, see $\S 4$.

Notes and references The idea of studying the Teichmüller space of onedimensional dynamical systems using invariant measures and Gibbs states is discussed in [Sul]; see also [Ca] and [SS]. A Weil-Petersson metric for $\mathcal{B}_{d}$ was introduced in [Mc2], motivating the present foundational considerations.

More on the dynamics of Blaschke products and inner functions can be found in $[\mathrm{Aa}]$, [Mar], [Neu], [Pom] and [PRS].

\section{Covering relations}

In this section we develop the theory of covering relations $f: S^{1} \rightarrow S^{1}$, and prove Theorem 1.1 along with other results regarding $\widehat{\operatorname{Exp}}_{d}\left(S^{1}\right)$ and the maximal measures $\mu_{f}$.

Marked covering maps. Let $\operatorname{Cov}_{d}\left(S^{1}\right)$ denote the space of degree $d$ topological covering maps $f: S^{1} \rightarrow S^{1}$, where $S^{1}=\mathbb{R} / \mathbb{Z}$. Its universal cover is the convex space $\operatorname{Cov}_{d}(\mathbb{R})$ of homeomorphisms $\tilde{f}: \mathbb{R} \rightarrow \mathbb{R}$ such that $\widetilde{f}(t+1)=d+\widetilde{f}(t)$. The group of rotations acts on $\operatorname{Cov}_{d}\left(S^{1}\right)$ by conjugation, yielding as quotient the moduli space

$$
\operatorname{MCov}_{d}\left(S^{1}\right)=S^{1} \backslash \operatorname{Cov}_{d}\left(S^{1}\right)=S^{1} \backslash\left(\operatorname{Cov}_{d}(\mathbb{R}) / \mathbb{Z}\right) .
$$

A marking for $f \in \operatorname{Cov}_{d}\left(S^{1}\right), d>1$, is a continuous degree one map $\phi$ : $S^{1} \rightarrow S^{1}$ satisfying the semiconjugacy condition

$$
\phi \circ f(t)=\phi \circ p_{d}(t) .
$$

A marking always exists, and satisfies

$$
\widetilde{\phi}(t)=\lim d^{-n} \widetilde{f}^{n}(t)
$$

for a suitable lift of $f$. If we replace $\widetilde{f}(t)$ by $\widetilde{f}(t)+1$, then $\phi(t)$ is replaced by $\phi(t)+1 /(d-1)$. Thus the Teichmüller space of marked covering maps $[f, \phi]$ is given by:

$$
\operatorname{TCov}_{d}\left(S^{1}\right)=S^{1} \backslash\left(\operatorname{Cov}^{d}(\mathbb{R}) /(d-1) \mathbb{Z}\right) .
$$


The mapping class group

$$
\operatorname{Mod}_{d} \cong \mathbb{Z} /(d-1) \cong \operatorname{Aut}\left(p_{d}\right)
$$

acts on this Teichmüller space by rotating the marking by $1 /(d-1)$, yielding moduli space as its quotient. Summarizing, for $d>1$ we have a commutative diagram

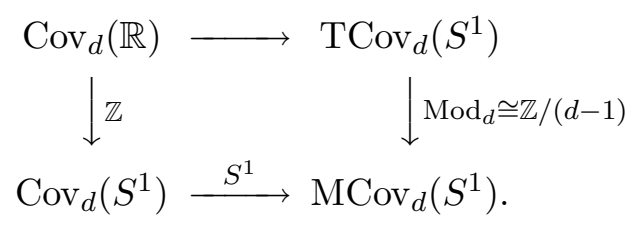

Note that $\left[p_{d}, \mathrm{id}\right]$ is fixed by the full group $\operatorname{Mod}_{d}$, and hence it determines an orbifold point in moduli space.

Relations. For $X=\mathbb{R}$ or $S^{1}$, let $\operatorname{Rel}(X)$ denote the space of closed subsets $f \subset X \times X$. We given $\operatorname{Rel}(X)$ the Hausdorff metric,

$$
D(f, g)=\inf \{r>0: B(f, r) \supset g \text { and } B(g, r) \supset f\},
$$

which can be infinite when $X=\mathbb{R}$. Bounded sets are compact in $\operatorname{Rel}(X)$; in particular, $\operatorname{Rel}\left(S^{1}\right)$ is compact.

We regard $f \in \operatorname{Rel}(X)$ as a relation, or multivalued map, defined on $E \subset X$ by

$$
f(E)=\{x \in X:(e, x) \in f \text { for some } e \in E\} .
$$

The graph of a relation is the original closed set $f \subset X \times X$. Composition is defined by $(f \circ g)(E)=f(g(E))$. Any ordinary function is a relation; so is its inverse.

Limits of homeomorphisms. For later use we record some basic properties of limits of homeomorphisms, $f \in \overline{\operatorname{Homeo}}(\mathbb{R}) \subset \operatorname{Rel}(\mathbb{R})$.

1. Any such $f$ is obtained from a monotone function by replacing its jump discontinuities with vertical segments. It graph is a rectifiable copy of $\mathbb{R}$ properly embedded in $\mathbb{R} \times \mathbb{R}$.

2. Although $\operatorname{Homeo}(\mathbb{R})$ is closed under composition, its closure is not. For example, if $f_{1}([a, b])=p$ and $f_{2}(p)=[a, b]$, then the graph of $f_{2} \circ f_{1}$ contains the square $[a, b] \times[a, b]$.

3. Let $\mu$ be a locally finite Borel measure on $\mathbb{R}$ without atoms. Then there is a well-defined pullback measure $f^{*}(\mu)$ characterized by:

$$
f^{*}(\mu)(E)=\mu(f(E)) .
$$


If $\lambda$ denotes Lebesgue measure on the real line, then $f^{*}(\lambda)$ agrees with the distributional derivative $m^{\prime}$ of any monotone function whose graph is contained in $f$.

4. Let $M(\mathbb{R})$ denote the space of locally finite measures on $\mathbb{R}$ with the weak topology. Then for any fixed $\mu \in M(\mathbb{R})$ without atoms, the map $\overline{\operatorname{Homeo}}(\mathbb{R}) \rightarrow M(\mathbb{R})$ given by $f \mapsto f^{*}(\mu)$ is continuous.

Covering relations. Now let

$$
\widehat{\operatorname{Cov}}_{d}(\mathbb{R})=\left\{\begin{array}{l}
f \in \overline{\operatorname{Homeo}}(\mathbb{R}): f(t+1)=f(t)+d, \\
\text { and } f^{-1} \text { is a continuous function. }
\end{array}\right\}
$$

We define the space of degree $d$ covering relations on the circle by

$$
\widehat{\operatorname{Cov}}_{d}\left(S^{1}\right)=\widehat{\operatorname{Cov}}_{d}(\mathbb{R}) / \mathbb{Z} .
$$

Note that a covering relation is allowed to be infinitely expanding (it can blow a point up to an interval), but not infinitely contracting (it cannot blow an interval down to a point).

Since $f^{-1}(t) \bmod 1$ is continuous and periodic, $f^{-1}$ is uniformly continuous, and thus the composition map

$$
\widehat{\operatorname{Cov}}_{d}\left(S^{1}\right) \times \widehat{\operatorname{Cov}}_{e}\left(S^{1}\right) \rightarrow \widehat{\operatorname{Cov}}_{d+e}\left(S^{1}\right)
$$

is well-defined and continuous. (This would fail if we allowed both infinite expansion and contraction.)

Sources and multiplicities. A covering relation $f=[\widetilde{f}] \in \widehat{\operatorname{Cov}}_{d}\left(S^{1}\right)$ determines an ordinary relation $f$ on the circle, by $f(t \bmod 1)=\widetilde{f}(t) \bmod 1$. But the lift $\widetilde{f}$ also contains multiplicity data, which is lost when we pass to the graph of $f$.

This data is encoded by an effective divisor $S=\sum m_{i} s_{i} \in \operatorname{Div}\left(S^{1}\right)$, where the sources $s_{i}$ are the points satisfying $f\left(s_{i}\right)=S^{1}$, and the multiplicities $m_{i}=\left[\left|\widetilde{f}\left(\widetilde{s}_{i}\right)\right|\right]$ are the integer parts of the lengths of the intervals $\widetilde{f}\left(\widetilde{s}_{i}\right)$, $s_{i}=\widetilde{s}_{i} \bmod 1$.

When $f$ has no sources, it is determined by its graph in $S^{1} \times S^{1}$. In general the graph of $f$ contains the graph of a unique covering relation $F$ without sources, possibly of lower degree. We have $\operatorname{deg}(S)+\operatorname{deg}(F)=$ $\operatorname{deg}(f)$, and the pair $(F, S)$ determines $f$ uniquely.

One can visualize $f=(F, S) \in \widehat{\operatorname{Cov}}_{d}\left(S^{1}\right)$ as a map which expands each source $s_{i}$ to the immersed interval $\left[a_{i}, m_{i}+b_{i}\right] \bmod 1$, where $F\left(s_{i}\right)=\left[a_{i}, b_{i}\right]$. 
A covering relation determines a pullback map for measures on $S^{1}$ by:

$$
f^{*}(\mu)=F^{*}(\mu)+\mu\left(S^{1}\right) \sum m_{i} \delta_{s_{i}}
$$

and $f^{*}$ determines $f$.

Markings and the maximal measure. Next we extend the notion of a marking to covering relations.

Theorem 2.1 For any $f \in \widehat{\operatorname{Cov}}_{d}(\mathbb{R}), d>1$, there is a unique $\phi \in \overline{\operatorname{Cov}}_{1}(\mathbb{R})$ such that

$$
\phi \circ f(x)=d \phi(x)
$$

for all $x \in \mathbb{R}$. Moreover $\phi$ depends continuously on $f$.

Here $\overline{\operatorname{Cov}}_{1}(\mathbb{R})$ is the closure of $\operatorname{Cov}_{1}(\mathbb{R})$ in $\operatorname{Rel}(\mathbb{R})$.

Proof. Let $\phi_{n}=d^{-n} f^{n}(x) \in \widehat{\operatorname{Cov}}_{1}(\mathbb{R})$. Since $f^{-1}$ is continuous, we have $\phi_{n} \in \widehat{\operatorname{Cov}}_{1}(\mathbb{R})$ and $\phi_{n}$ is a continuous function of $n$. Note that by periodicity, the relation $s=\phi_{1}(t)=d^{-1} f(t)$ is a bounded distance from the diagonal $s=t$. Thus for all $n$ and $t$, the compact sets $f^{n}(t)$ and $d^{-1} f^{n+1}(t)$ have Hausdorff distance $O(1)$. It follows that

$$
D\left(\phi_{n}, \phi_{n+1}\right)=O\left(d^{-n}\right)
$$

in the Hausdorff metric on $\operatorname{Rel}(\mathbb{R})$, and hence $\phi_{n}$ converges to the required relation $\phi \in \overline{\operatorname{Cov}}_{1}(\mathbb{R})$. Since the convergence is uniform, $\phi$ also depends continuously on $f$.

For uniqueness, suppose another $\psi \in \overline{\operatorname{Cov}}_{1}(\mathbb{R})$ also satisfies $\psi \circ f=d \psi$; then repeating the argument above, we find $\phi=\lim d^{-n} \psi f^{n}=\psi$.

Corollary 2.2 For any $f \in \widehat{\operatorname{Cov}}_{d}\left(S^{1}\right), d>1$, there is a $\phi \in \overline{\operatorname{Cov}}_{1}\left(S^{1}\right)$, unique up to the action of $\operatorname{Mod}_{d}$, such that $\phi \circ f=p_{d} \circ \phi$ in $\overline{\operatorname{Cov}}_{d}\left(S^{1}\right)$.

We refer to such a pair $(f, \phi)$ as a marked covering relation.

Corollary 2.3 For every $f \in \widehat{\operatorname{Cov}}_{d}\left(S^{1}\right), d>1$, there exists a unique probability measure $\mu_{f}$ on $S^{1}$ such that $f^{*}\left(\mu_{f}\right)=d \mu_{f}$. Moreover $\mu_{f}$ depends continuously on $f$, and

$$
\mu_{f}=\lim d^{-n}\left(f^{n}\right)^{*}(\alpha)
$$

for any probability measure $\alpha$ on $S^{1}$. 
We refer to $\mu_{f}$ as the maximal measure, since it generalizes the measure of maximum entropy for $f \in \operatorname{Cov}_{d}\left(S^{1}\right)$.

Proof. The proof follows the same lines as the proof of Theorem 2.1. In fact $\mu_{f}=\phi^{*}(\lambda)$, where $\lambda$ is Lebesgue measure on $S^{1}$; since $\phi$ varies continuously with $f$, so does $\mu_{f}$.

Moduli spaces. We define moduli and Teichmüller spaces of covering relations by replacing $\operatorname{Cov}_{d}$ with $\widehat{\operatorname{Cov}}_{d}$ in equations (2.1) and (2.2). The preceding results show that $\widehat{\mathrm{Cov}}_{d}\left(S^{1}\right)$ is naturally identified with the space of marked covering relations.

Expanding maps. We now focus on expanding relations. Let

$$
\operatorname{Exp}_{d}\left(S^{1}\right)=\left\{f \in \operatorname{Cov}_{d}\left(S^{1}\right): f_{*}(\lambda)=\lambda\right\}
$$

be the space of covering maps of degree $d$ that preserve Lebesgue measure $\lambda$ on the circle.

Any $f \in \operatorname{Exp}_{d}\left(S^{1}\right)$ is expanding; it satisfies

$$
\left.|I|<\mid f^{-1} f(I)\right)|=| f(I) \mid
$$

on any interval where $f \mid I$ is injective. Conversely, any smooth map $g: S^{1} \rightarrow$ $S^{1}$ with $\inf \left|g^{\prime}(t)\right|>1$ is smoothly conjugate to a map $f \in \operatorname{Exp}_{d}\left(S^{1}\right)$ (cf. [Krz], [Sac], [SS, Cor. 4]).

For relations, the condition $f_{*}(\lambda)=\lambda$ defines a sublocus $\widehat{\operatorname{Exp}}_{d}\left(S^{1}\right) \subset$ $\widehat{\operatorname{Cov}}_{d}\left(S^{1}\right)$, and similar loci $\widehat{\operatorname{Exp}}_{d}\left(S^{1}\right)$ and $T \widehat{\operatorname{Exp}}_{d}\left(S^{1}\right)$ in moduli and Teichmüller spaces. The space $\widehat{\operatorname{Exp}}_{d}(\mathbb{R}) \subset \widehat{\operatorname{Cov}}_{d}(\mathbb{R})$ is determined by the condition

$$
\sum_{i=1}^{d}\left(T_{i} \circ \widetilde{f}\right)_{*}(\lambda)=\lambda,
$$

where $T_{i}(x)=x+i$; this insures that $\widetilde{f}$ covers a relation $f \in \widehat{\operatorname{Exp}}_{d}\left(S^{1}\right)$.

Theorem 2.4 The space $\widehat{\operatorname{Exp}}_{d}\left(S^{1}\right)$ is compact.

Proof. The expanding condition implies the inverse of any lift $\widetilde{f} \in \widehat{\operatorname{Cov}}_{d}(\mathbb{R})$ is a continuous function satisfying the Lipschitz condition

$$
\left|\tilde{f}^{-1}(s)-\tilde{f}^{-1}(t)\right| \leq|s-t| .
$$

The space of such functions, modulo translation, is compact, and the condition $f_{*}(\lambda)=\lambda$ is preserved in the limit. 
Corollary 2.5 The Teichmüller and moduli spaces $\widehat{\operatorname{TExp}}\left(S^{1}\right)$ and $\widehat{\operatorname{MExp}}\left(S^{1}\right)$ are also compact.

Invariant measures. Every $(f, \phi) \in \mathrm{T} \widehat{\operatorname{Exp}}\left(S^{1}\right)$ determines an invariant measure

$$
\nu_{(f, \phi)}=\phi_{*}(\lambda)
$$

for the action of $p_{d}$ on $S^{1}$. We can now prove our main result: that the map

$$
\nu: \widehat{\operatorname{Exp}}_{d}\left(S^{1}\right) \rightarrow M_{d}\left(S^{1}\right)
$$

is a homeomorphism.

Proof of Theorem 1.1. The measure $\nu_{(f, \phi)}$ depends continuously on $(f, \phi)$ by Theorem 2.1. So it suffices to show $\nu$ is bijective. To this end, suppose $\alpha \in M\left(S^{1}\right)$ is an invariant probability measure for $p_{d}$. Let $\widetilde{\alpha}$ be the lift of $\alpha$ to a $\mathbb{Z}$-invariant measure on $\mathbb{R}$. By $p_{d}$-invariance, we have

$$
\widetilde{\alpha}(d I) \geq \widetilde{\alpha}(I)
$$

for all intervals $I$. Let $m: \mathbb{R} \rightarrow \mathbb{R}$ be a monotone function satisfying $m^{\prime}=\alpha$ as a distribution, and let $\widetilde{\phi} \in \overline{\operatorname{Homeo}}(\mathbb{R})$ be the unique limit of homeomorphisms such that $\widetilde{\phi}^{-1}$ contains the graph of $m$. Then $\widetilde{\phi}_{*}(\lambda)=\widetilde{\alpha}$, and $\widetilde{\phi}(t+1)=\widetilde{\phi}(t)+1$ by periodicity of $\alpha$; in other words, we have $\widetilde{\phi} \in$ $\overline{\operatorname{Cov}}_{1}(\mathbb{R})$.

Consider the monotone, proper relation

$$
F(t)=\widetilde{\phi}^{-1}(d \widetilde{\phi}(t)) \in \operatorname{Rel}(\mathbb{R}) .
$$

Clearly $F(t+1)=F(t)+d$. We will show that $F$ contains the graph of a unique $\tilde{f} \in \widehat{\operatorname{Exp}}_{d}(\mathbb{R})$.

First suppose $\widetilde{\alpha}$ has no atoms. We claim that

$$
F^{-1}(t)=\widetilde{\phi}^{-1}\left(d^{-1} \widetilde{\phi}(t)\right)
$$

is a monotone continuous function. To see this, note that when $\alpha$ has no atoms the map $m=\widetilde{\phi}^{-1}$ is monotone continuous, and so the only danger arises $\widetilde{\phi}(x)=I$ is an interval instead of a point. But in this case we have

$$
\lambda\left(F^{-1}(x)\right)=\lambda\left(\widetilde{\phi}^{-1}\left(d^{-1} I\right)\right)=\widetilde{\alpha}\left(d^{-1} I\right) \leq \widetilde{\alpha}(I)=\lambda(\{x\})=0,
$$

by equation $(2.5)$, and thus $F^{-1}(x)$ is still a single point. Therefore $F^{-1}$ is monotone continuous. 
By similar reasoning, we have $\phi \circ F(x)=d \phi(x)$ for all $x$. It follows that $\tilde{f}=F$ belongs to $\widehat{\operatorname{Cov}}_{d}(\mathbb{R})$, and thus $f=([\widetilde{f}],[\widetilde{\phi}])$ belongs to $\widehat{\mathrm{TCov}}_{d}\left(S^{1}\right)$.

We claim $f$ preserves Lebesgue measure. Indeed, since $\alpha$ is invariant under $p_{d}$, we have

$$
\widetilde{\left(p_{d}\right)_{*}(\alpha)}=\sum_{i=1}^{d}(d \cdot t+i)_{*}(\widetilde{\alpha})=\widetilde{\alpha} .
$$

But $\widetilde{\alpha}$ has no atoms, so this implies

$$
\begin{aligned}
\widetilde{f_{*}(\lambda)} & =\sum_{i=1}^{d}(\widetilde{f}(x)+i)_{*}(\lambda)=\phi_{*}^{-1}\left(\sum_{i=1}^{d}(d x+i)_{*} \phi_{*}(\lambda)\right) \\
& =\phi_{*}^{-1}\left(\sum_{i=1}^{d}(d x+i)_{*}(\widetilde{\alpha})\right)=\phi_{*}^{-1}(\widetilde{\alpha})=\lambda .
\end{aligned}
$$

Thus $(f, \phi)$ belongs to $\widehat{\operatorname{Exp}}_{d}\left(S^{1}\right)$, and by construction it satisfies $\nu_{(f, \phi)}=\alpha$; and it is the unique such map up to conjugation by a rotation, since the solution to $m^{\prime}=\alpha$ is unique up to translation.

Now suppose $\widetilde{\alpha}$ has atoms. Choose $t \in \mathbb{R}$ such that $\widetilde{\alpha}(\{t\})>0$. Then $I=\widetilde{\phi}^{-1}(t)$ is an interval of length $\lambda(I)=\widetilde{\alpha}(t)>0$. Since $\alpha$ is invariant under $p_{d}, t \bmod 1$ is a periodic point for $p_{d}$, and hence $\widetilde{\alpha}(d \cdot t)=\widetilde{\alpha}(t)$. Thus $J=\widetilde{\phi}^{-1}(d \cdot t)$ is an interval of the same length as $I$, and hence we have $I \times J \subset F \subset \mathbb{R} \times \mathbb{R}$. Let us replace this square with its diagonal, to obtain the graph of a translation from $I$ to $J$. Carrying out the same procedure for every atom of $\alpha$, we again obtain a relation $\widetilde{f} \subset F$ whose inverse is a continuous function. This diagonalized version of $F$ is the unique $\widetilde{f} \in \widehat{\operatorname{Exp}}_{d}(\mathbb{R})$ whose graph is contained in $F$.

By construction, pushforward under $f=[\widetilde{f}]$ preserves the atomic part of $\alpha$, and by the preceding argument it also preserves the non-atomic part. Thus we have $(f, \phi) \in \operatorname{TExp}_{d}\left(S^{1}\right)$, and again (up to rotation) this is the unique expanding relation with $\nu_{(f, \phi)}=\alpha$.

Example: an interval exchange. Let $\nu$ be the unique invariant probability measure for $p_{2}(t)=2 t \bmod 1$ supported on the periodic cycle $P=$ $(1,2,3,4) / 5 \subset S^{1}$. Let $(f, \phi) \in \operatorname{Exp}_{2}\left(S^{1}\right)$ be the unique marked expanding relation (up to rotation) with $\nu_{(f, \phi)}=\nu$. Then $f$ has four points of discontinuity, dividing the circle into open intervals which are permuted in the pattern (1342) by $f$. The map $\phi$ blows these intervals down to the points 
of $P$, and blows their endpoints up to the intervals forming $S^{1}-P$. Consequently the graph of the relation $\phi^{-1}(d(\phi(t)))$ contains 4 squares; replacing these by their diagonals yields the graph of $f$. See Figure 1.

In this example $f$ is a periodic interval exchange transformation: it isometrically permutes finitely many open intervals, which fill the circle.

Graph of $\phi$

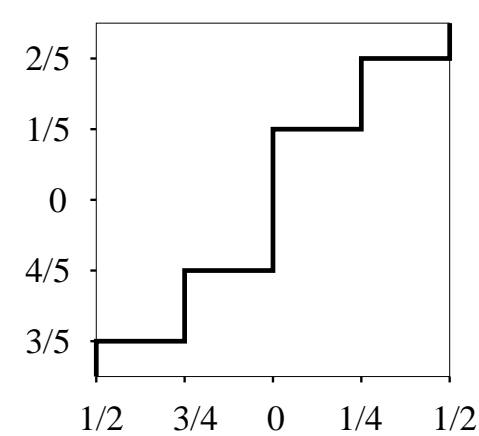

Graph of $f$

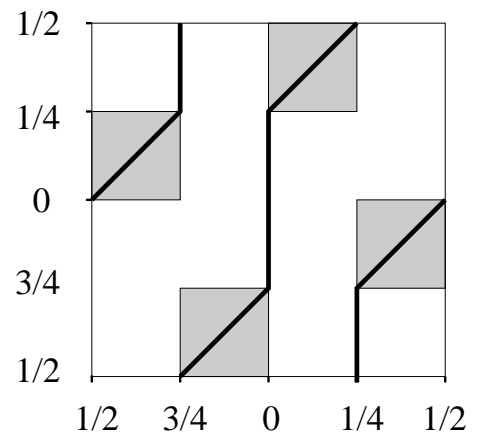

Figure 1. The graphs of $\phi$ and $f$, with the latter superimposed on the relation $\phi^{-1} \circ p_{d} \circ \phi$.

Properties of measures and maps. Here are some readily verified relations between $f \in \widehat{\operatorname{Exp}}_{d}\left(S^{1}\right)$ and the measures $\mu=\mu_{f}$ and $\nu=\nu_{f, \phi}$ (for any marking $\phi$ ).

1. We have $f \in \operatorname{Exp}_{d}\left(S^{1}\right) \Longleftrightarrow \nu$ has full support and no atoms $\Longleftrightarrow \mu$ has full support and no atoms $\Longleftrightarrow \phi$ is a homeomorphism.

2. The measure $\nu$ has finite support $\Longleftrightarrow \mu$ has finite support $\Longleftrightarrow f$ is a periodic interval exchange transformation.

3. Write $S^{1}-\operatorname{supp} \nu=\bigcup I_{i}$ as a union of disjoint open intervals. Then the number of sources of $f$ is given by

$$
\operatorname{deg}(S)=\sum\left[d\left|I_{i}\right|\right]
$$

where $[x]=\sup \{n \in \mathbb{Z}: n \leq x\}$. Level sets of the sum above give the stratification of $M_{d}\left(S^{1}\right)$ corresponding to the stratification of $\widehat{\operatorname{Exp}}_{d}\left(S^{1}\right)$ by $\operatorname{deg}(F)$.

4. The measure $\nu$ has no atoms iff for any open interval $I$, we have $f^{n}(I)=S^{1}$ for some $n>0$ ( $f$ is locally eventually onto). 
It is also routine to verify:

Proposition 2.6 If $f=((F, S), \phi)$ with $F \in \operatorname{Exp}_{e}\left(S^{1}\right)$, and $F$ is not a periodic interval exchange transformation, then

1. $K=\operatorname{supp} \nu_{(f, \phi)}$ is either a Cantor set or the full circle,

2. $\psi=\phi^{-1}: S^{1} \rightarrow S^{1}$ is continuous and surjective,

3. $\psi: S^{1} \rightarrow S^{1}$ collapses the intervals forming the complement of $K$ to points, and is otherwise injective; and

4. We have a surjective semiconjugacy

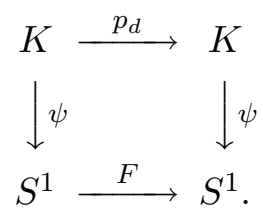

Topology of the compactification. To conclude, we use the homeomorphism $\nu$ to show:

Theorem 2.7 The space $\operatorname{TExp}_{d}\left(S^{1}\right)$ is dense in its compactification $\widehat{\operatorname{Txp}}_{d}\left(S^{1}\right)$, and both spaces are contractible.

Lemma 2.8 Invariant measures without atoms are dense in $M_{d}\left(S^{1}\right)$.

Proof. Let $x$ be one of the countably many periodic points for $p_{d}: S^{1} \rightarrow S^{1}$. Let $\beta(x)$ denote the unique invariant probability measure supported on the forward orbit of $x$, and for $k>0$ let

$$
M_{k}(x)=\left\{\alpha \in M\left(S^{1}, p_{d}\right): \alpha(x) \geq 1 / k\right\} .
$$

Any atom of an invariant measure must be supported at a periodic point, and hence the set of invariant measures with atoms coincides with the countable union $\bigcup_{x, k} M_{k}(x)$. It is easy to see that the periodic points

$$
x_{n}=x+1 /\left(d^{n}-1\right)
$$

satisfy $\beta\left(x_{n}\right) \rightarrow \beta(x)$ as $n \rightarrow \infty$, and thus any $\alpha \in M_{k}(x)$ can be approximated by measures with atoms along the orbit of $x_{n}$ instead of along the orbit of $x$. Consequently $M_{k}(x)$ is nowhere dense in $M_{d}\left(S^{1}\right)$. By the Baire category theorem, $\bigcup_{x, k} M_{k}(x)$ is also nowhere dense. 
Proof of Theorem 2.7. Any measure $\alpha \in M\left(S^{1}, p_{d}\right)$ without atoms is a limit of the invariant measures $(1-t) \alpha+t \lambda$ which also have full support, and hence have the form $\nu_{(f, \phi)}$ for some $f \in \operatorname{Exp}_{d}\left(S^{1}\right)$. Thus $\operatorname{Exp}_{d}\left(S^{1}\right)$ is dense. Contractibility follows from convexity of the corresponding spaces of measures.

\section{Blaschke products}

In this section we identify $S^{1}=\mathbb{R} / \mathbb{Z}$ with the unit circle in the complex plane, using the coordinate $z=\exp (2 \pi i t)$, and study expanding maps on $S^{1}$ with a holomorphic extension to the unit disk $\Delta=\{z:|z|<1\}$. In particular we prove Theorems 1.2 and 1.4.

Blaschke products. For $d>1$, let $\mathcal{B}_{d}$ denote the space of Blaschke products $f: \bar{\Delta} \rightarrow \bar{\Delta}$ of the form

$$
f(z)=z \prod_{1}^{d-1}\left(\frac{z-a_{i}}{1-\bar{a}_{i} z}\right)
$$

with $a_{i} \in \Delta$.

It is well-known that any $f \in \mathcal{B}_{d}$ preserves Lebesgue measure on $S^{1}$. Indeed, any rational function fixing 0 and $\infty$ satisfies $f_{*}(d z / z)=d z / z$, by residue considerations, and $(d z / z) \mid S^{1}$ gives Lebesgue measure (cf. [Mar]). Conversely, any $f \in \operatorname{Exp}_{d}\left(S^{1}\right)$ with a holomorphic extension to the disk is conjugate by a rotation to an element of $\mathcal{B}_{d}$.

Algebraic compactification. Since $f$ is determined by the unordered list of points $\left(a_{i}\right)_{1}^{d-1}, \mathcal{B}_{d}$ can be naturally identified with the symmetric product $\Delta^{(d-1)}$. Taking the closure in $\mathbb{C}^{(d-1)}$, we obtain the algebraic compactification

$$
\overline{\mathcal{B}}_{d}=\bar{\Delta}^{(d-1)}=\bigsqcup_{e=1}^{d-1} \Delta^{(e-1)} \times\left(S^{1}\right)^{(d-e)} .
$$

We will identify a point $\left(a_{i}\right) \in \overline{\mathcal{B}}_{d}$ with the covering relation $f=(F, S) \in$ $\widehat{\operatorname{Exp}}_{d}\left(S^{1}\right)$ given by

$$
F(z)=z \prod_{\left|a_{i}\right|<1}\left(\frac{z-a_{i}}{1-\bar{a}_{i} z}\right) \cdot \prod_{\left|a_{i}\right|=1}\left(-a_{i}\right)
$$

and

$$
S=\sum_{\left|a_{i}\right|=1} a_{i} \in \operatorname{Div}\left(S^{1}\right)
$$


If $f_{n} \in \mathcal{B}_{d}$ converges to $\left(F, S=\sum m_{i} s_{i}\right) \in \partial \overline{\mathcal{B}}_{d}$, then their graphs satisfy

$$
\operatorname{gr}\left(f_{n}\right) \rightarrow \operatorname{gr}(F)+\sum m_{i}\left(\left\{s_{i}\right\} \times \widehat{\mathbb{C}}\right)
$$

as divisors of degree $(1, d)$ on $\widehat{\mathbb{C}} \times \widehat{\mathbb{C}}$. In particular the graphs in $S^{1} \times S^{1}$ (and their lifts to $\mathbb{R} \times \mathbb{R}$ ) converge, and hence the natural inclusion

$$
\overline{\mathcal{B}}_{d} \subset \widehat{\operatorname{Exp}}_{d}\left(S^{1}\right)
$$

is continuous.

Markings. Since $\overline{\mathcal{B}}_{d}$ is simply-connected, there is a unique choice of marking $\phi_{f}$ which varies continuously with $f \in \overline{\mathcal{B}}_{d}$ and satisfies $\phi_{f}(z)=z$ when $f(z)=z^{d}$. The map $f \mapsto\left(f, \phi_{f}\right)$ gives an embedding

$$
\overline{\mathcal{B}}_{d} \rightarrow \widehat{\mathrm{Exp}}_{d}\left(S^{1}\right) \text {. }
$$

Its image is $\operatorname{Mod}_{d}$-invariant, and $\operatorname{Mod}_{d}$ acts on $\overline{\mathcal{B}}_{d}$ by

$$
\left(a_{i}\right) \mapsto\left(\zeta a_{i}\right)
$$

where $\zeta^{d-1}=1$.

Fixed points. Note that the marking $\phi_{f}$ picks out a distinguished fixedpoint $z_{f}=\phi_{f}^{-1}(1)$ for any $f \in \mathcal{B}_{d}$, or more generally for any $f=(F, S) \in \overline{\mathcal{B}}_{d}$ with $F \neq$ id. In the latter case $z_{f}$ is either a source or a fixed point of $F$.

Here is a direct description of the distinguished source in the case where $F(z)=-z$. Suppose $d$ is even. By (3.1) the sources of $f=(F, S)$ (repeated according to their multiplicities) satisfy $F^{\prime}(0)=-1=\prod\left(-s_{i}\right)=-\prod s_{i}$; therefore, they can be uniquely ordered so they admit lifts $\widetilde{s}_{i} \in \mathbb{R}$ satisfying

$$
\widetilde{s}_{1} \leq \widetilde{s}_{2} \leq \cdots \leq \widetilde{s}_{d}=\widetilde{s}_{1}+1 \text { and } \sum_{1}^{d-1} \widetilde{s}_{i}=0 .
$$

The canonical source is then given by $z_{f}=s_{d / 2}$. Similarly, when $d$ is odd the lifts can be chosen so that $\widetilde{s}_{1} / 2+\widetilde{s}_{2}+\cdots+\widetilde{s}_{d-1}+\widetilde{s}_{d} / 2=0$; then $z_{f}=s_{(d+1) / 2}$.

Embedding and strata. We can now restate and prove Theorems 1.2 and 1.4 .

Theorem 3.1 The map $\overline{\mathcal{B}}_{d} \rightarrow \widehat{\operatorname{TExp}}_{d}\left(S^{1}\right) \cong M_{d}\left(S^{1}\right)$ is an embedding.

Proof. This follows from the preceding discussion together with Theorem 1.1. 
Theorem 3.2 For any $f=(F, S) \in \overline{\mathcal{B}}_{d}$ with $\operatorname{deg}(F)=e$, we have

$$
\text { H. } \operatorname{dim}\left(\operatorname{supp} \nu_{f}\right)=\frac{\log e}{\log d} \text {. }
$$

Proof. If $e=1$ then $F$ is a rotation; otherwise, $F \mid S^{1}$ is topologically conjugate to $z \mapsto z^{e}$. Since topological conjugacy does not change the support of $\nu$, in the latter case we can replace $F$ by $z^{e}$. Thus can assume $\lambda(F(I))=e \lambda(I)$ whenever $F \mid I$ is injective. It follows that $\nu_{f}\left(p_{d}(I)\right)=e \nu(I)$ whenever $p_{d} \mid I$ is injective.

Now let $I$ be any interval centered on a point of $\operatorname{supp} \nu_{f}$. Let $n=$ $[\log |I| / \log d]$. Then $p_{d}^{n}$ maps $I$ injectively to an interval $J$ of definite length and definite $\nu$-measure. Since $|J|=d^{n}|I|$ and $\nu_{f}(J)=e^{n} \nu(I)$, we have

$$
\nu_{f}(I) \asymp|I|^{\log e / \log d},
$$

which implies H. $\operatorname{dim}(\operatorname{supp} \nu)=\log e / \log d(\mathrm{cf}$. [Fal, §4.1]).

Notes. The algebraic completion of $\mathcal{B}_{d}$ agrees with its closure in the compactification $\overline{\mathrm{Rat}_{d}} \cong \mathbb{P}^{2 d+1}$ of the space of degree $d$ rational maps $f: \widehat{\mathbb{C}} \rightarrow \widehat{\mathbb{C}}$, considered in $[\mathrm{D} 1, \S 1]$. In $\overline{\text { Rat }_{d}}$ the limiting objects are effective divisors $f \subset \widehat{\mathbb{C}} \times \widehat{\mathbb{C}}$ of degree $(1, d)$, which can be interpreted dynamically as algebraic correspondences. In this framework, our definition of $\mu_{f}$ is consistent with that given in [D1].

It would be interesting to determine the closure of $\mathcal{B}_{d}$ in the compactified moduli spaces of rational maps provided by [Sil] and [D2].

\section{Polynomials and harmonic measure}

In this section we show that certain polynomials provide geometric models for covering relations, their invariant measures and their markings. For background on Julia sets, external angles and harmonic measure, see e.g. [Bro], [DH], [CG], [Mil2] and [Mil3].

Polynomials. Let $P(z)=z^{d}+b_{1} z^{d-1}+\cdots+b_{d}$ be a monic polynomial of degree $d>1$. Its filled Julia set is the space of bounded orbits, defined by

$$
K(P)=\left\{z \in \mathbb{C}: \sup _{n>0}\left|P^{n}(z)\right|<\infty\right\} .
$$

Its Julia set is $J(P)=\partial K(P)$.

Assume that $K(P)$ is locally connected, that $P(0)=0$, and that $z=0$ belongs to a component $U_{0}(P)$ of the interior of $K(P)$. Let $K_{0}(P)$ denote the closed disk $\overline{U_{0}(P)}$, and let $J_{0}(P)=\partial K_{0}(P)$. Then $P$ uniquely determines: 
- A measure of maximal entropy $\mu_{P}$ on $J(P)$, equal to the hitting measure for Brownian motion initiated at $z=\infty$,

- A harmonic measure $\nu_{P}$ on $J_{0}(P)$, equal to the hitting measure for Brownian motion initiated at $z=0$;

- A retraction $\rho: K(P) \rightarrow K_{0}(P)$ which is locally constant outside $K_{0}(P)$;

- A Riemann mapping

$$
\alpha:(\widehat{\mathbb{C}}-\bar{\Delta}, \infty) \rightarrow(\widehat{\mathbb{C}}-K(P), \infty),
$$

normalized so $(\alpha)^{\prime}(\infty)>0$, and satisfying $\alpha\left(z^{d}\right)=P(\alpha(z))$;

- A continuous extension

$$
\alpha: S^{1} \rightarrow J(P)
$$

(which exists by local connectivity), labeling points in the Julia set by external angles;

- A Riemann mapping

$$
\beta:(\Delta, 0) \rightarrow\left(U_{0}(P), 0\right),
$$

normalized by $\beta^{\prime}(0)>0$, and also extending continuously to $S^{1}$;

- A Blaschke product $F:(\Delta, 0) \rightarrow(\Delta, 0)$ of degree $1 \leq e \leq d$, preserving Lebesgue measure on the circle, and transported by $\beta$ to $P \mid U_{0}(P)$;

- A divisor $S_{P} \in \operatorname{Div}\left(J_{0}(P)\right)$ of degree $(d-e)$, given by the sum (with multiplicities) of $\rho(c)$ over the $(d-e)$ critical points of $P$ that are not in $U_{0}(P)$; and

- A divisor $S \in \operatorname{Div}\left(S^{1}\right)$ given by $S=\beta^{*}\left(S_{P}\right)$.

We can now formulate a more precise statement of Theorem 1.5.

Theorem 4.1 Let $f=(F, S) \in \widehat{\operatorname{Exp}}_{d}\left(S^{1}\right)$ be determined by $P$ as above. Then:

1. The measure $\mu_{f}$ corresponds, under $\beta$, to the retraction of the maximal measure $\rho_{*}\left(\mu_{P}\right)$; 
2. The relation $\phi(z)=\alpha^{-1} \circ \rho^{-1} \circ \beta(z)$ gives a marking for $f$; and

3. The measure $\nu_{(f, \phi)}$ corresponds, under $\alpha$, to the harmonic measure $\nu_{P}$ on $J_{0}(P)$.

Proof. It is easily verified that $\phi \circ f=p_{d} \circ \phi$, and thus $\phi$ is a marking for $f$. The assertions on measures then follow from the fact $\mu_{P}, \nu_{P}, \mu_{f}$ and $\nu_{(f, \phi)}$ are the images of Lebesgue measure on $S^{1}$ under $\alpha_{*}, \beta_{*}, \phi^{*}$ and $\phi_{*}$ respectively.

Example 1: Quasicircles. We have $J(P)=J_{0}(P) \Longleftrightarrow \operatorname{deg}(F)=d \Longleftrightarrow$ $\left|P^{\prime}(0)\right|<1$ and all critical points of $P$ lies in the immediate basin $U_{0}(P) \Longleftrightarrow$ $J(P)$ is a quasicircle. In this case $\phi$ is simply the topological conjugacy between $z^{d}$ and $F(z)$ given geometrically by the boundary correspondence across $J(P)$.

Example 2: The golden mean Siegel disk. Let $P(z)=\lambda z+z^{2}$, where $\lambda=\exp (2 \pi \gamma)$ and $\gamma=(1+\sqrt{5}) / 2$. Then $U_{0}(P)$ is a Siegel disk, and $K(P)$ is locally connected [Pet]. In this case $(F, S)=(\lambda z,-\lambda)$. The external rays landing on the Siegel disk determine a Cantor set $K \subset S^{1}$ of Hausdorff dimension zero.

The Siegel disk is the lower large component of the interior of $K(P)$, shown in gray in Figure 2; the hyperbolic convex hull of the Cantor set $K$ is shown at the right.

Each complementary arc of $K$ corresponds to a component of $K(P)-$ $K_{0}(P)$. The Cantor set $K$ itself is the set of points that never escape from $[a, a+1 / 2] \subset S^{1}$ under iteration of $p_{d}$, where $a$ and $a+1 / 2$ are the external angles of the critical point of $P$. Explicitly, $a=0 . a_{1} a_{2} a_{3} \ldots=$ $0.010110101101101 \ldots$ in base 2 ; we have $a_{i}=0$ if $i \gamma \bmod 1 \in[0,2-\gamma]$, and $a_{i}=1$ otherwise.
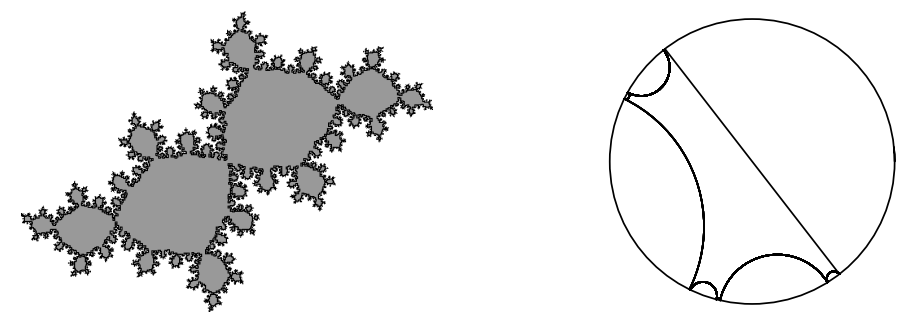

Figure 2. A quadratic Siegel disk and a Cantor set of dimension zero. 
Example 3: Hyperbolic centers in the Mandelbrot set. Let $Q(z)=$ $z^{2}+c$ be a quadratic polynomial whose critical point $z=0$ has period $q$, and let $P(z)=Q^{q}(z)$. Then $U_{0}(P)$ is the immediate basin of attraction of $z=0$ for $Q$; we have $F(z)=z^{2}$ (up to conjugation by a rotation); and $\operatorname{deg} S=2^{q}-2$. The set $K=\operatorname{supp} \nu_{f}$ consists of the angles of external rays landing in $\partial U_{0}(P)$, and satisfies

$$
\text { H. } \operatorname{dim}(K)=\log \operatorname{deg}(F) / \log \operatorname{deg}(P)=1 / q .
$$

Example 4: A cubic with a superattracting fixed point. Let $P(z)=$ $3 z^{2}+4 z^{3}$. Then $P$ has a superattracting fixed point at $z=0$, with immediate basin $U_{0}(P)$; and the other critical point $c=-1 / 2$ lands on the repelling fixed point $z=1 / 4 \in \partial U_{0}(P)$ after one iterate. In this case $f=(F, S)=$ $\left(z^{2},-1\right)$, and $K=\operatorname{supp} \nu_{f}$ is a copy of the standard middle-thirds Cantor set, reduced mod 1 ; it satisfies

$$
\text { H. } \operatorname{dim}(K)=\log 2 / \log 3 .
$$

The Julia set $J(P)$ and the convex hull of $K$ are shown in Figure 3.
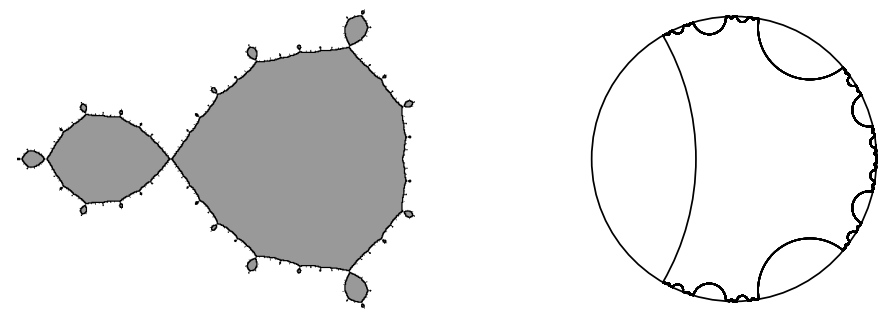

Figure 3. A superattracting cubic basin and a Cantor set of dimension $\log 2 / \log 3$.

Failure of realizability. For $d=2$, a given $f \in \overline{\mathcal{B}}_{d}$ can only be realized by a polynomial conjugate to $P(z)=\lambda z+z^{2}$, with $\lambda=f^{\prime}(0)$. But this realization may not exist: for example, when $\lambda \in S^{1}$ is very well-approximated by roots of unity, the 'Cremer point' $z=0$ belongs to the Julia set of $P$, so there is no domain $U_{0}(P)$ to work with.

For higher degrees the realizing polynomial $P$, even when it exists, is generally not unique; the behavior of the critical points outside of $U_{0}(P)$ contributes additional moduli.

Notes. Related material on the golden mean Siegel disk, cubic polynomials, and laminations can be found in [Mc1], [Mil1] and [Ke]. 


\section{References}

[Aa] J. Aaronson. A remark on the exactness of inner functions. J. London Math. Soc. 23(1981), 469-474.

[Bon] F. Bonahon. The geometry of Teichmüller space via geodesic currents. Invent. math. 92(1988), 139-162.

[Bro] H. Brolin. Invariant sets under iteration of rational functions. Ark. Math. 6(1965), 103-144.

[CG] L. Carleson and T. Gamelin. Complex Dynamics. Springer-Verlag, 1993.

[Ca] E. E. Cawley. The Teichmüller space of an Anosov diffeomorphism of $T^{2}$. Invent. math. 112(1993), 351-376.

[D1] L. DeMarco. Iteration at the boundary of the space of rational maps. Duke Math. J. 130(2005), 169-197.

[D2] L. DeMarco. The moduli space of quadratic rational maps. J. Amer. Math. Soc. 20(2007), 321-355.

[DH] A. Douady and J. Hubbard. Étude dynamique des polynômes complexes. Pub. Math. d'Orsay 84-2, 85-4, 1985.

[Fal] K. J. Falconer. Fractal Geometry. Wiley, 2003.

[FLP] A. Fathi, F. Laudenbach, and V. Poénaru. Travaux de Thurston sur les surfaces. Astérisque, vol. 66-67, 1979.

[Fra] J. Franks. Anosov diffeomorphisms. In Global Analysis, Proc. Sympos. Pure Math., Vol. XIV, pages 61-93, 1970.

[Gol] L. Goldberg. Fixed points of polynomial maps I. Rotation subsets of the circles. Ann. Sci. Éc. Norm. Sup. 25(1992), 679-685.

[Ke] K. Keller. Invariant Factors, Julia Equivalences and the (Abstract) Mandelbrot Set, volume 1732 of Lecture Notes in Mathematics. Springer-Verlag, 2000.

[Krz] K. Krzyzewsky. Some results on expanding mappings. Astérisque 50(1977), 205-218. 
[Mar] N. F. G. Martin. On finite Blaschke products whose restrictions to the unit circle are exact endomorphisms. Bull. London Math. Soc. 15(1983), 343-348.

[Mc1] C. McMullen. Self-similarity of Siegel disks and the Hausdorff dimension of Julia sets. Acta Math. 180(1998), 247-292.

[Mc2] C. McMullen. Thermodynamics, dimension and the Weil-Petersson metric. Inv. math. 173(2008), 365-425.

[Mil1] J. Milnor. Remarks on iterated cubic maps. Experiment. Math. 1(1992), 5-24.

[Mil2] J. Milnor. Dynamics in One Complex Variable: Introductory Lectures. Vieweg, 1999.

[Mil3] J. Milnor. Periodic points, external rays and the Mandelbrot set: an expository account. In Géométrie complexe et systèmes dynamiques (Orsay, 1995), pages 277-333. Astérisque, vol. 261, 2000.

[Neu] J. H. Neuwirth. Ergodicity of some mappings of the circle and the line. Israel J. Math. 31(1978), 359-367.

[Pet] C. L. Petersen. Local connectivity of some Julia sets containing a circle with an irrational rotation. Acta Math. 177(1996), 163-224.

[Pom] Ch. Pommerenke. On ergodic properties of inner functions. Math. Ann. 256(1981), 43-50.

[PRS] E. R. Pujals, L. Robert, and M. Shub. Expanding maps of the circle rerevisited: positive Lyapunov exponents in a rich family. Ergodic Theory Dynam. Systems 26(2006), 1931-1937.

[Sac] R. Sacksteder. The measures invariant under an expanding map. In Géométrie différentielle, volume 392 of Lecture Notes in Mathematics, pages 179-194. Springer, 1981.

[Sh] M. Shub. Endomorphisms of compact differentiable manifolds. Amer. J. Math. 91(1969), 175-199.

[SS] M. Shub and D. Sullivan. Expanding endomorphisms of the circle revisited. Ergodic Theory Dynam. Systems 5(1985), 285-289.

[Sil] J. Silverman. The space of rational maps on $\mathbf{P}^{\mathbf{1}}$. Duke Math. J. 94(1998), 41-77. 
[Sul] D. Sullivan. Quasiconformal homeomorphisms in dynamics, topology and geometry. In Proceedings of the International Conference of Mathematicians, pages 1216-1228. Amer. Math. Soc., 1986.

Mathematics Department

HARVARD UNIVERSITY

1 OXFORD ST

CAmbridge, MA 02138-2901 